\title{
Hydrodynamic cavitation damage in water at elevated temperatures
}

\author{
Matevž Dular ${ }^{1 *}$
}

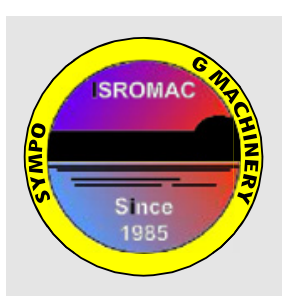

ISROMAC 2016

International

Symposium on Transport

Phenomena and Dynamics of

Rotating Machinery

Hawaii, Honolulu

April 10-15, 2016

\begin{abstract}
In the present study we show experimental campaign where cavitation erosion in water at different temperatures was investigated. In contrary to other studies, where cavitation is generated by ultrasound, we employed hydrodynamic cavitation, which more closely resembles the conditions in applications - it is known that the results obtained by ultrasonic cavitation can be misleading. The tests were performed in a radial flow test-section, which can generate very aggressive type of cavitation. Polished aluminum samples were used to investigate the damage. Temperatures in the range between 30 and $100^{\circ} \mathrm{C}$ were investigated. We found out that the temperature of the water significantly influences the cavitation aggressiveness -maximum aggressiveness was found at $60^{\circ} \mathrm{C}$. In the last part of the work two theories were developed and tested. Micro-jet approach correctly predicted the trend but the influence of the temperature was marginal. On the other hand, the theory of the spherical bubble collapse with consideration of thermodynamic effects of cavitation produced a very good agreement to the experiments.
\end{abstract}

\section{Keywords}

cavitation; erosion; thermodynamic effect; model; thermodynamic parameter

\footnotetext{
${ }^{1}$ Faculty of Mechanical Engineering, University of Ljubljana, Slovenia

*Corresponding author: matevz.dular@fs.uni-lj.si
}

\section{INTRODUCTION}

Cavitation is a phenomenon characterized by vapor generation and condensation in high-speed liquid flows. It frequently occurs in industrial configurations such as rotating machinery, injectors, and other hydraulic devices. Most of the time it is accompanied by effects like vibrations, increase of hydrodynamic drag, changes in the flow hydrodynamics, noise, erosion, light effects such as sonoluminescence, and also thermal effects [1, 2].

Thermodynamic effects become significant only when the critical-point temperature is close to the operating temperature of the fluid, as in the case of cryogenic fluids [3]. Therefore, the understanding and the prediction cavitation effects in such cases is crucial in many applications; for example the turbopumps for liquid hydrogen LH2 and oxygen LOX in space launcher engines need to have an inducer rotor installed upstream from the main impellers, in order to achieve high suction performance [4]. The inducer is designed to operate in moderate cavitating conditions, hence a minimum pressure level in the tanks must be ensured, in order to avoid the occurrence of large sheet cavities on the blades, which are often associated with large-scale instabilities. Particularity well known is the failure of the Japanese $\mathrm{H}$-II rocket due to rotating cavitation in the LH2 turbopump [5].
The new generation of rocket engines will also feature the possibility of re-ignition while in orbit, hence long term operation of LH2 and LOX turbo-pumps under cavitation conditions is becoming an issue.

A wide range of studies related to various aspects of the cavitation erosion problem - bubble dynamics, model development, CFD prediction, material testing etc. have been performed in the past [6-12]. They all aim at improving the physical understanding of the phenomenon. Yet it seems, that with every new step we find new phenomena and physical background, which prove that cavitation erosion process is even more complex and its prediction even more elusive [13].

The effects of medium temperature on the aggressiveness of cavitation erosion were studied as early as 1960's ans 70's when Garcia \& Hammitt [14], Young \& Johnston [15] and Plesset [16] conducted vibratory tests. They have concluded that the decrease in the damage observed at higher temperatures can be attributed to either the increase in vapor pressure or the fact that the condensation driven collapse of a bubble at a higher temperature is slower, since more heat needs to be conducted into the surrounding fluid as a result of higher vapor density - when the temperature and pressure of the uncondensed vapor are raised, they arrest the bubble collapse, decrease collapse pressures and consequently damage.

More recently the effects of medium and its temperature 
on acoustic cavitation aggressiveness were studied by Hatori $[17,18]$. While they show that both play significant role in the process, few conclusions on the physical background of the measured results are provided.

In contrary to other studies, where cavitation is generated by ultrasound, we employed hydrodynamic cavitation, which more closely resembles the conditions in space propulsion applications. Cavitation aggressiveness on aluminum samples in water with different temperatures (from low to significant level of thermodynamic effects) was observed. In the discussion we derive a bubble dynamics model, which explains the dependency between the temperature of the medium (its thermodynamic parameter ?][19]) and cavitation aggressiveness.

The present study is a step towards evaluation of erosion in cryogenic liquids under a scope of the continuous work for the European Space Agency (ESA).

\section{EXPERIMENT}

Cavitation tests were performed in a small cavitation tunnel at the Laboratory for Water and Turbine Machines, University of Ljubljana. The geometry was adopted from the facility at the LEGI Grenoble [9, 20].

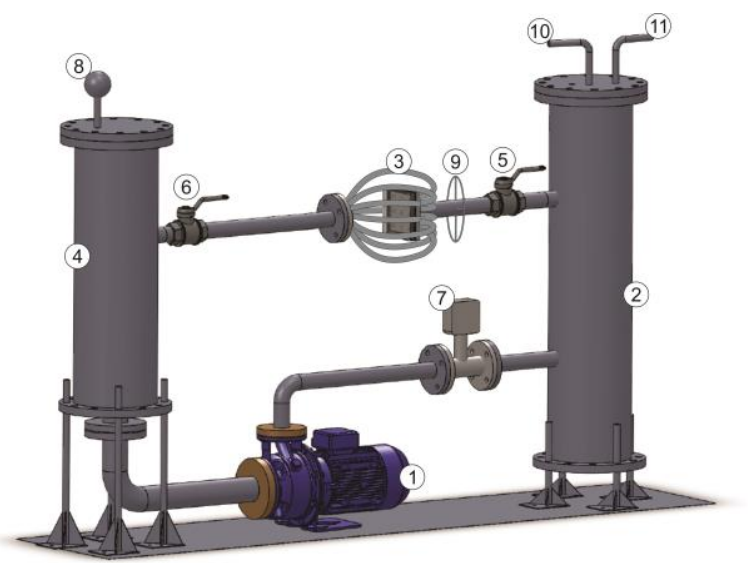

Figure 1. The test-rig.

A $4.5 \mathrm{~kW}$ pump (1) enables the variation of the rotation frequency in order to set the flow rate. Downstream of the pump, a partially filled tank (2) is installed for water heating and for damping the periodical flow rate and pressure fluctuations. Cavitation and its effects are observed in a test section (3). The water enters the section axially through the nozzle and exits it by 8 flexible pipes. The tank further downstream (4) is used for cooling of the circulation water - a secondary cooling water loop is installed in it. The valves (5) and (6) enable easy and fast disconnection of the test section from the main loop. The flow rate is measured by an electromagnetic flow meter (7) ABB ProcessMaster 300 (DN 40) with a $2 \%$ uncertainty on measurements. Temperature is obtained with a type $\mathrm{K}$ thermocouple (8). The reference pressure is measured $35 \mathrm{~mm}$ upstream from the test-section by an ABB 266AST pressure transducer (9) - the uncertainty of the measurements is $8 \mathrm{mbar}$. The pressure in the test rig is adjusted in the partially filled tank (2) connected to a compressor (10) and a vacuum pump (11). The precisions of the pressure, velocity and temperature measurements result in a mean uncertainty of $3 \%$ for the cavitation number.

\subsection{Test-section}

To ensure a highly aggressive cavitation conditions one needs to develop a test section where i) the pressure recuperation is rapid and ii) cavitation clouds implode in the vicinity of the specimen. Both conditions are easily achieved in the so-called radial jet testsection shown schematically in Fig. 2.

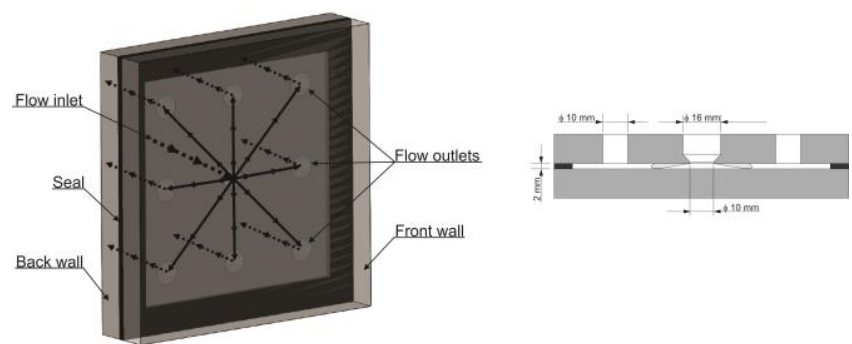

Figure 2. Test section design with indicated general flow pattern.

The flow enters the section axially at a high velocity through a nozzle with a diameter of $10 \mathrm{~mm}$. It then forced to turn at an angle of $90^{\circ}$, which makes it cavitate. The radius of curvature of the feed nozzle exit is $0.75 \mathrm{~mm}$. The gap between the front and back plates is $2 \mathrm{~mm}$ wide. As the fluid moves radially outwards between the plates the pressure recuperates rapidly and cavitation zone abruptly closes. The flow exits the section axially through 8 holes in the back plate. The position of the specimen center lies at a radial distance of $22.2 \mathrm{~mm}$ from the center of the feed nozzle.

\subsection{Specimens}

Specimens (Fig. 3) were manufactured out of aluminum. The diameter of the surface which was exposed to cavitation is $30 \mathrm{~mm}$. 

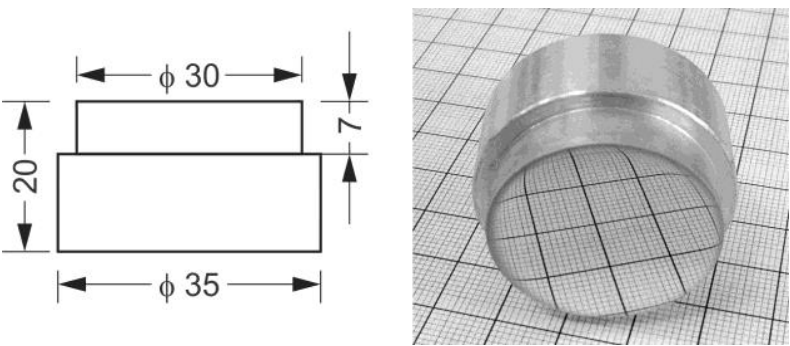

Figure 3. Dimensions of the specimen (in $\mathrm{mm}$ ) and its picture prior to the exposure to cavitation.

The surface was polished down to 10 $\mathrm{m}$. The surface hardness was measured to be $65 \mathrm{HV}$.

\subsection{Observation of cavitation}

For the purpose cavitation observation the front plate of the test-section was replaced with a transparent one. The idea of this test was to obtain some general characteristics of cavitation behavior inside the section.

High speed camera Fastec Imaging HiSpec4 2G mono was used for image acquisition. Figure 4 shows images of cavitation in the test section recorded at a frame rate of 50000 frames per second (only every 10th image is shown in Fig. 4). Images of cavitation were recorded at a resolution of $176 \times 86$ pixels.
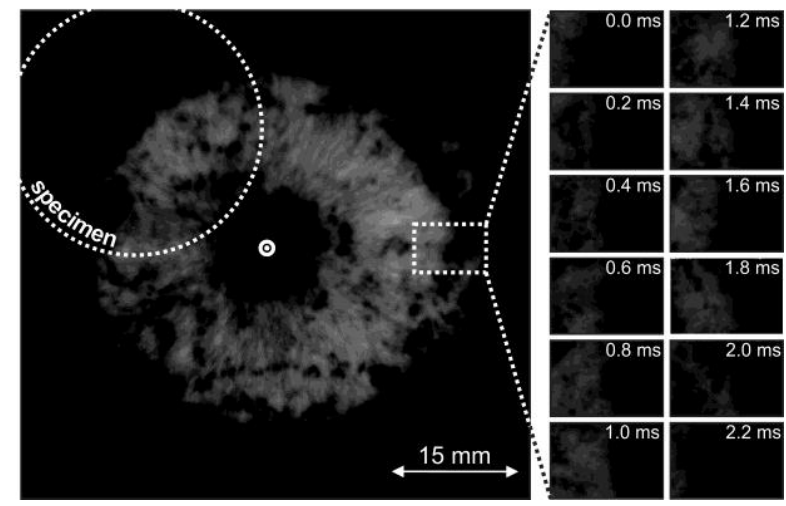

Figure 4. A single image (frontal view) and a high speed sequence showing cavitation in the test-section $\left(T=30^{\circ} \mathrm{C}\right.$, $\left.\mathrm{v}=20.5 \mathrm{~m} / \mathrm{s}, \mathrm{p}^{\infty}=4.305 \mathrm{bar}\right)$. The position of the specimen is shown by dashed line.

Our results relate well to the observations in the original design from LEGI laboratory [21]. As mentioned, the water begins to cavitate at a $90^{\circ}$ bend as it enters the gap between the plates. Cavitation clouds separate from the attached cavity and travel radially outwards to a higher pressure region where they collapse - roughly 20 to $25 \mathrm{~mm}$ from the center of the plate. We expect the highest erosion rate in the region of cavitation cloud collapse - near the outer perimeter of cavitation zone. A circle in Fig. 4 shows the position of the specimen.
Article Title - 3

The test parameters included 8 conditions where the water temperature was varied between 30 and $100^{\circ} \mathrm{C}$ and specimens were exposed to cavitation for a period 120 minutes. For cases of water temperature of 30 and $40^{\circ} \mathrm{C}$, four additional measurements were made where the specimens were exposed to cavitation for only of 30 and 60 minutes.

\section{EROSION EVALUATION}

After the exposure to cavitation the specimen was photographed with a TESA-VISIO $300 \mathrm{GL}$ DCC system. To photograph the entire surface of the specimen about 80 images were taken, each covering the area of 5.5 by $4 \mathrm{~mm}$ at a pixel size of approximately $7 \mu \mathrm{m}$.

The images can be stacked together to obtain a high resolution image of the whole specimen surface (Fig. 5).

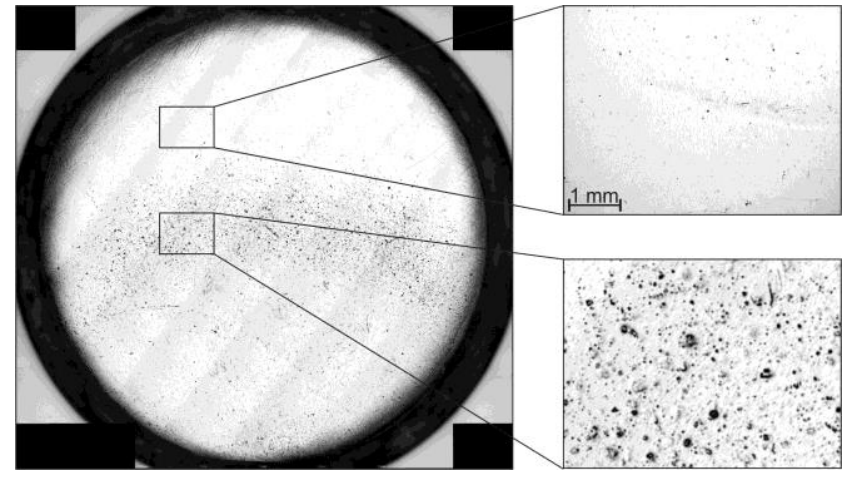

Figure 5. High resolution image of specimen surface, which has been exposed to cavitation for 120 minutes in water at $\mathrm{T}=30^{\circ} \mathrm{C}$.

Comparing the two individual images the damaged area can clearly be seen. As expected the damage follows a circle with the center in the middle of the test-section - the maximum damage lies at a radius of about $22 \mathrm{~mm}$ - the same as the closure region of cavitation (Fig. 4).

The evaluation followed the pit-counting technique [7, 10]. Pits are recognized as the darker regions in an image, while the brighter area is assumed to be undamaged surface. The pitcount method gives a distribution of the number and the area of the pits and consequently, the distribution of the magnitude of cavitation erosion on the surface.

Figure 6 shows a typical result of evaluation (again for the case where specimen was exposed to cavitation for 120 minutes in water at $\mathrm{T}=30^{\circ} \mathrm{C}$ ).

\subsection{Test conditions}




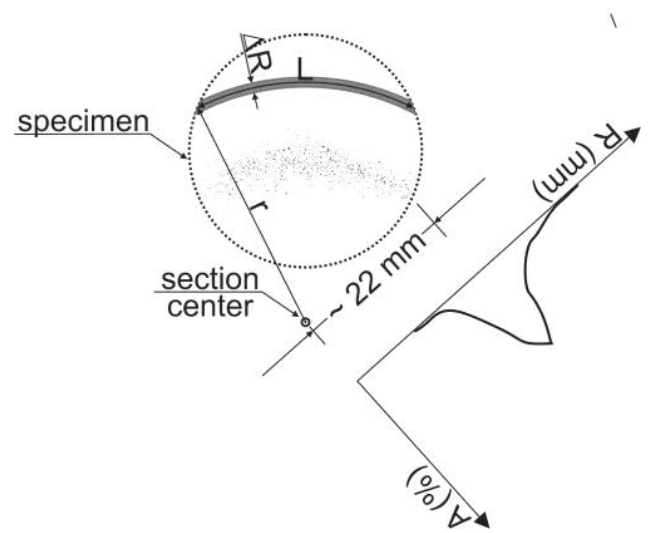

Figure 6. A typical result of pit-count evaluation (Water temperature is $30^{\circ} \mathrm{C}$, time of exposure is $120 \mathrm{~min}$ ).

One can clearly see that the damage is concentrated along a circular pattern at a radial distance of approximately $22 \mathrm{~mm}$ from the center of the test-section. Figure 6 also shows how the results were averaged according to the radial distance from the center of the section. One can describe the averaging by:

$$
A(r)=\frac{1}{L(r) \cdot \Delta R} \cdot \sum_{r=7.2 m m}^{r=37.2 m m} A_{p i t}(r)
$$

where $A_{\text {pit }}$ is the damaged area within an evaluated band (shown as gray area), $L$ is the evaluated arch length (depending of the distance from the section center) and $\Delta R$ is the width of the evaluated band (about $0.1 \mathrm{~mm}$ ). $A(r)$ represents the part of the area covered by pits and is given in \%. The definition of the cavitation aggressiveness is unified among the researchers - most commonly it is related to the pit volume or the deformation energy needed to form a pit. It was shown in several studies [23-27] that the measurement of the surface of the pit correlates well to the pit volume, the damage energy and consequently to cavitation aggressiveness. Our present observations of the surfaces did not reveal significant dependence between the topology of the pit and the medium temperature; hence the area $A(r)$ can be used as an indicator of cavitation aggressiveness.

\section{RESULTS}

The results are presented in diagrams showing the percentage of the damaged surface as a function of radial distance from the center of the section (Fig. 6 and Eqn. 1).

First tests where the samples were exposed to cavitation at a low temperature $\left(30\right.$ and $\left.40^{\circ} \mathrm{C}\right)$ and for different lengths of time are shown and discussed (Fig. 7).
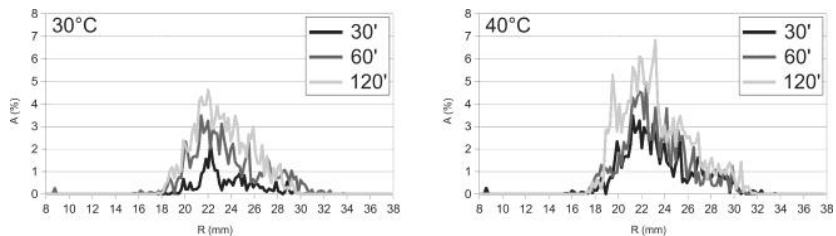

Figure 7. Damage distributions for tests at 30 and $40^{\circ} \mathrm{C}$ for exposure times of 30,60 and 120 minutes.

These tests were performed to determine the most suitable time of exposure and also to evaluate the method. One can see that at a maximum about $5-7 \%$ of the surface was covered by pits after 120 minutes of exposure. Such a value is well placed as it lies in a range where significant number of pits is accumulated for further statistical evaluation, but there is also not significant overlapping of the damage, which could distort the results [7].

One can also see that the damage increases approximately linearly with time, which corresponds to our previous experiments [28]. The maximum of the damage lies roughly 22 $\mathrm{mm}$ from the center of the section (in the middle of the specimen), which coincides with the cavity closure line (Fig. 4).

Figure 8 shows the results of measurements where the specimens were exposed to cavitation for a period of 120 minutes at water temperatures between 30 and $100^{\circ} \mathrm{C}$.
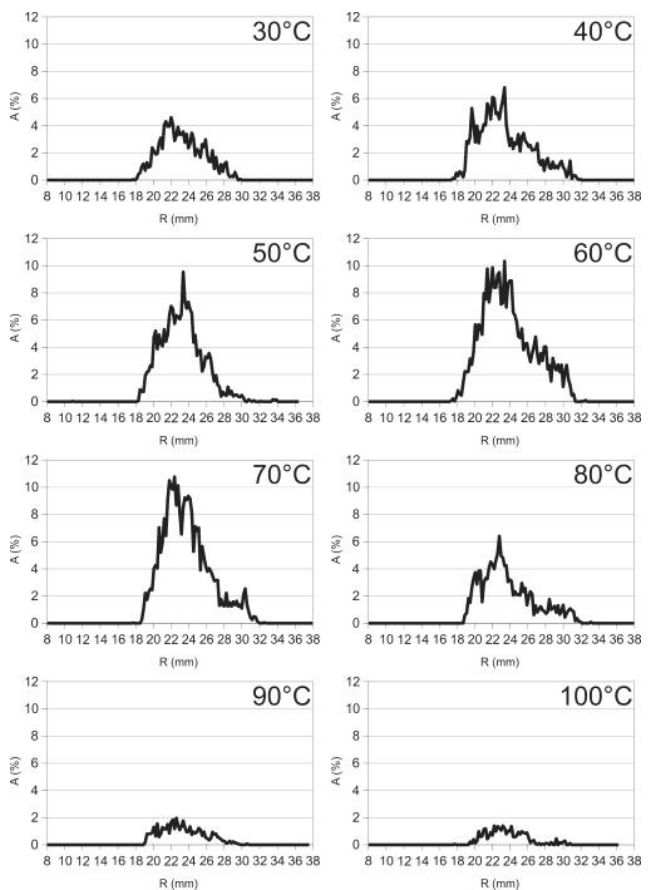

Figure 8. Damage distributions for tests at temperatures from 30 to $100^{\circ} \mathrm{C}$ for exposure time of 120 minutes.

One can clearly see that cavitation aggressiveness is strongly dependent on the temperature of the medium. As the temperature was increased from $30^{\circ} \mathrm{C}$ also the damage extent rose. The maximum is achieved at around $60^{\circ} \mathrm{C}$. The cavitation remains aggressive for water of $70^{\circ} \mathrm{C}$, then it sharply drops to level below the one at $30^{\circ} \mathrm{C}$. The effected region did not change significantly between the tests - most of the damage is concentrated around the cavity closure line at $R=22 \mathrm{~mm}$.

\section{DISCUSSION}

Studies agree that cavitation structures carry a significant amount of potential energy and can, at their collapse, emit pressure waves of magnitude of several MPa [29]. Yet it seems that the macroscopic cavitation cloud collapse itself cannot be a 
direct cause of erosion as its energy is not enough concentrated. Currently the most widely accepted explanation of the phenomenon is that the potential energy contained in a macro-cavity transformed into the radiation of acoustic pressure waves, and further on into the erosive power contained in the micro-scale cavitation structures or single bubbles that collapse in the vicinity of the material boundaries $[30,31]$.

Two theories describe the last stages of life of a micro-scale cavitation structure - the micro-jet and the spherical microbubble collapse [32]. Since the scientific community is divided between the two, we have used both approaches for explaining the physics behind our experimental results.

\subsection{Micro-jet}

If we assume that the bubble will collapse in the form of a micro-jet we can estimate its velocity according to the theory by Plesset \& Chapmann [33] and to experimental and numerical work by Chahine [32]:

$$
v_{m j}=k \cdot \sqrt{\frac{p_{\infty}-p_{v}}{\rho}} .
$$

$\mathrm{k}$ is a constant, which was determined to be $\mathrm{k}=4.6$ by Chahine [32]. It is common to obtain velocities of the micro-jet in the order of several hundred $\mathrm{m} / \mathrm{s}$. The pressure induced on the solid material is the water hammer pressure reduced by the efficiency of the impact, which lies at $60 \%$ [32]:

$$
p_{w h}=0.6 \cdot c \cdot \rho \cdot v_{m j},
$$

where $c$ is the liquid sonic velocity. One can easily calculate the great erosive potential of such collapse. To take the effect of the temperature into an account we only need to consider that the sonic velocity and the density of the liquid will change with temperature. Figure 9 shows the dependence of the water hammer pressure as a function of temperature compared to experimental results of measured damage (the part of the specimen surface which was covered by pits Aint). We postulate that there is a relationship between the magnitude of water hammer pressure and damage [13, 34]. Since we do not have the means to determine the pressure difference in Eqn. 2 we present the result in its nondimensional form: (if we assume that the pressure difference is independent of the temperature, then the prediction is only a function of liquid sonic velocity and liquid density, which are both temperature dependent).

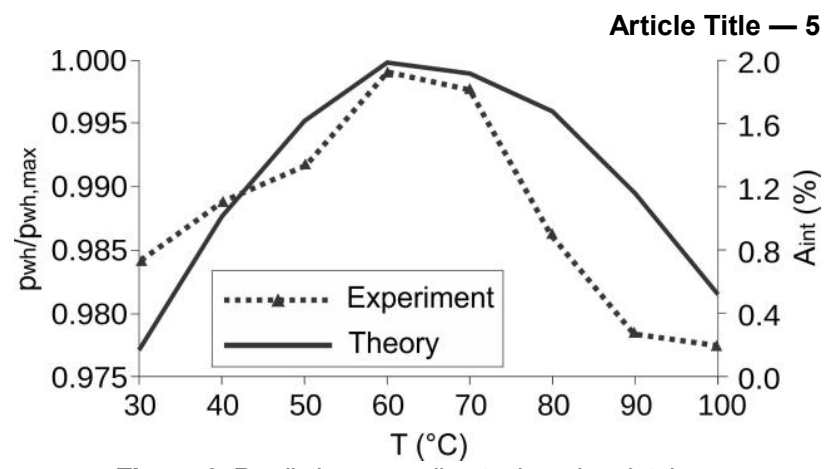

Figure 9. Prediction according to the micro-jet theory.

Although we see that the micro-jet theory predicts the maximum to lie at $60^{\circ} \mathrm{C}$, what agrees with experiments, the predicted temperature influence on cavitation aggressiveness is insignificant - only about $3 \%$. Such a small variation in the predicted water hammer pressure cannot explain the obvious and dependence of cavitation aggressiveness on the medium temperature. On the basis of these results we can deem the micro-jet approach as questionable or even false in the present case.

\subsection{Spherical collapse}

The second widely accepted mechanism that takes place in creating a pit is the shock wave, which is emitted at a spherical collapse of an even smaller bubble. The pressure field, in terms of the distance from the bubble center and the time pcs(r,t), can be determined by solving the Reyleigh-Plesset equation, which, for the case of fluids with considerable themodynamic effect, needs to be modified [1, 19]:

$$
\begin{aligned}
& \rho_{l}\left(R \ddot{R}+\frac{3}{2} \dot{R}^{2}\right)+\Sigma \dot{R} \sqrt{t} \rho_{l}= \\
& \quad=p_{v}\left(T_{\infty}\right)-p_{\infty}+p_{g 0}\left(\frac{R_{0}}{R}\right)^{3 y}-\frac{2 S}{R}-4 \mu \frac{\dot{R}}{R}
\end{aligned}
$$

where:

$$
\Sigma=\frac{\left(\rho_{v} L\right)^{2}}{\rho_{l}^{2} c_{p l} T_{\infty} \sqrt{\alpha_{l}}}
$$

is the thermodynamic parameter [19] and it depends on the liquid temperature. Here $T_{\infty}$ is the test temperature, $\rho_{v}$ is the vapor density, $\rho_{I}$ is the liquid density, $L$ is the evaporative latent heat, $c_{p l}$ is the constant pressure specific heat of the liquid and $\alpha_{l}$ is the thermal diffusivity of the liquid.

The pressure emitted at collapse can be calculated as [35]:

$$
p_{s c}(r, t)=p_{\infty}+\rho_{l}\left[\ddot{R} \frac{R^{2}}{r}+2 \dot{R}^{2}\left(\frac{R}{r}-\frac{R^{4}}{4 r^{4}}\right)\right] .
$$

The results put the maximal pressure in an order of several hundred $\mathrm{MPa}$ or even $\mathrm{GPa}$ - again one can appreciate the high pressure and erosive potential of such collapse.

By investigating several boundary conditions we concluded that 
the choice of the initial bubble size and the ambient pressure evolution do not influence the general (non-dimensional) outcome of the calculation - although the bubble dynamics and shock wave magnitudes change significantly, the predicted dependency of the shock wave magnitude on the temperature does not change. Figure 10 shows the dynamics of cavitation bubble (its radius) in time as a function of the temperature. A bubble with an initial radius of 2 ? $\mathrm{m}$ was subjected to an oscillatory pressure field with amplitude. A constant pressure amplitude $f p^{\infty}, \max -p v(T)=1.4$ bar and frequency of $25 \mathrm{kHz}$ was used (this way constant cavitation conditions (constant cavitation number) in terms of the temperature were assured).
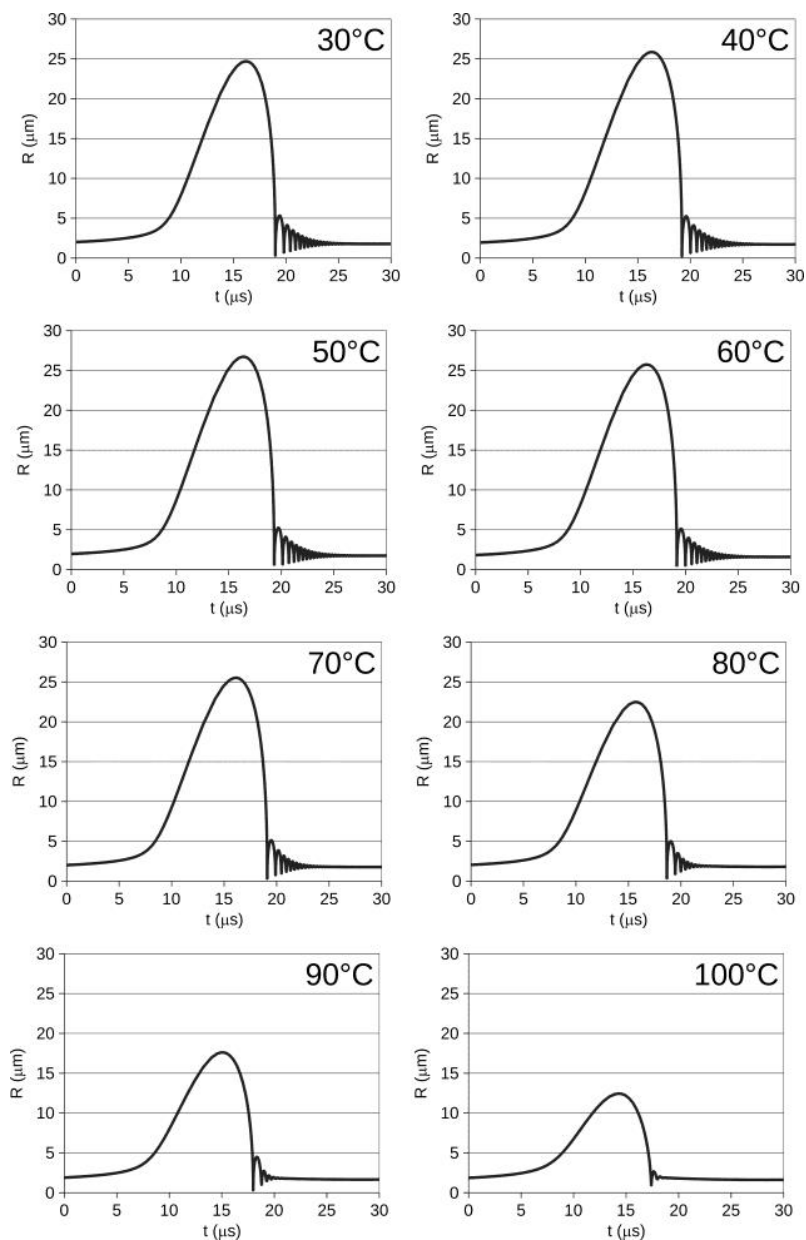

Figure 10. Predicted bubble radius as a function of time for temperatures between 30 and $100^{\circ} \mathrm{C}$.

We can see that the collapse occurs roughly between 17 and $20 \mu$ s after the bubble is exposed to the oscillatory pressure field. The bubble reaches the greatest size in the case at $50^{\circ} \mathrm{C}$ - about $27 \mu \mathrm{m}$, compared to, for example, only $12 \mu \mathrm{m}$ for the case of $100^{\circ} \mathrm{C}$. It is important to notice that the time taken for the collapse (from the instant when the bubble reaches its maximal size to the time of the first rebound) is almost the same to all temperatures - about $3 \mu \mathrm{s}$. Hence, the velocity of the collapse strongly depends on the temperature of the medium. Equation 6 states that the faster the collapse, the greater will the shock wave be.
Figure 11 shows the prediction of the shock wave amplitude as a function of temperature together with the measured integral damage (Aint) - again (as for the micro-jet approach presented in Fig. 9) we use a non-dimensional scale for the magnitude of the shock wave. A secondary $x$-axis gives the values of the thermodynamic parameter $\Sigma$ (Eqn. 5). For the sake of comparison we again assume that a relationship between the magnitude of the shockwave and damage exists [13, 34].

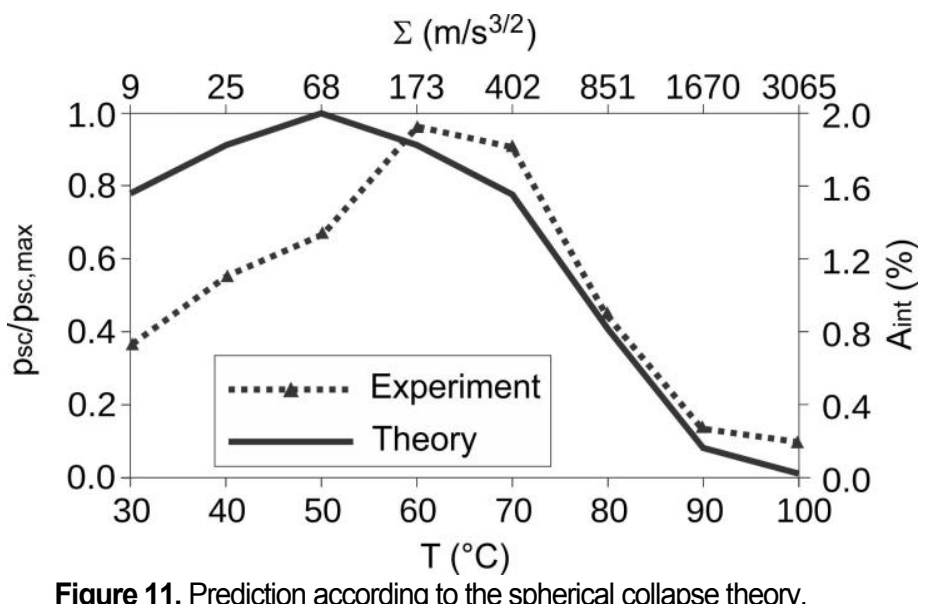

When the spherical bubble collapse theory with the addition of thermodynamic effects is considered the predicted trend lies much closer to the experimental one. A considerable influence can be seen at higher temperatures $\left(T>60^{\circ} \mathrm{C}\right)$ where the thermodynamic effects play a major role - according to Hattori [18] this is above $\Sigma>100 \mathrm{~m} / \mathrm{s}^{3 / 2}$.

The maximal magnitude of the shockwave is predicted at $50^{\circ} \mathrm{C}$, which somewhat differs from the maximum of measured erosion $\left(60^{\circ} \mathrm{C}\right)$. Also the prediction does not comply well with the experiment at lower temperatures, although the trend is correct. Nevertheless we conclude that the spherical bubble collapse theory with the addition of thermodynamic effects is a valuable tool for the prediction of cavitation aggressiveness in thermosensible fluids.

Interestingly the presented theory also complies with the measurements by Hattori et al. [17] who have shown that acoustically generated cavitation erosion in liquid nitrogen is marginal - the thermodynamic parameter of LN2 lies in the range of $\Sigma=3 \cdot 10^{5} \mathrm{~m} / \mathrm{s}^{3 / 2}$ - looking at the diagram in Fig. 11 one can indeed predict very gentle cavitation.

\section{CONCLUSIONS}

Measurements of hydrodynamic cavitation erosion in water at different temperatures were performed. In addition two models (micro-jet and spherical bubble collapse) were developed and tested against the measurements. The following conclusions can be drawn:

- As in the case of ultrasonic cavitation, in hydrodynamic cavitation the thermal effect on erosion aggressiveness needs to be considered when $\Sigma>100$ $\mathrm{m} / \mathrm{s}^{3 / 2}$. 
- Micro-jet theory correctly predicts the trend but the influence of the temperature is marginal.

- The theory of the spherical bubble collapse with consideration of thermodynamic effects of cavitation produced a very good agreement to the experiments.

- The theory of the spherical bubble collapse with consideration of thermodynamic effects also agrees with measurements in LN2 by Hattori et al. [17].

\section{ACKNOWLEDGMENTS}

The work was performed in the scope of the project "Cavitation in Thermosensible Fluids" financially supported by the European Space Agency (ESA).

\section{REFERENCES}

${ }^{[1]}$ M. Dular, O. Coutier-Delgosha, Thermodynamic effects during the growth and collapse of a single cavitation bubble, J. Fluid Mech. 736 (2013), 44-66.

[2] M. Petkovšek, M. Dular, IR measurements of the thermodynamic effects in cavitating flowOriginal Research Article, International Journal of Heat and Fluid Flow 44 (2013), 756-763.

${ }^{[3]}$ H.A. Stahl, A.J. Stepanoff, Thermodynamic aspects of cavitation in centrifugal pumps, ASME J. Basic Eng. 78 (1956), 1691-1693.

${ }^{[4]}$ K. Kamijo, H. Yamada, N. Sakazume, S. Warashina, Developmental History of Liquid Oxygen Turbopumps for the LE-7 Engine, Trans. Japan Soc. Aero. Space Sci. 44(145) (2001), 155-163.

${ }^{[5]}$ R. Sekita, A. Watanabel, K. Hirata, T. Imoto, Lessons learned from $\mathrm{H}-2$ failure and enhancement of $\mathrm{H}-2 \mathrm{a}$ project, Acta Astronautica 48 (5-12) (2001), 431-438.

[6] Lauterborn W, Bolle H. Experimental investigations of cavitation-bubble collapse in the neighbourhood of a solid boundary. Journal of Fluid Mechanics 1975; 72:391-399.

${ }^{[7}$ M. Dular, B. Bachert, B. Stoffel, B. Sirok, Relationship between cavitation structures and cavitation damage. Wear 257 (2004) 1176-1184.

${ }^{[8]}$ F. Pereira, F. Avellan, J.M. Dupont, Prediction of Cavitation Erosion: an Energy Approach, ASME J. Fluids Eng 120 (4) (1998) 719-727.

[9] J.P. Franc, Incubation Time and Cavitation Erosion Rate of Work-Hardening Materials, J. Fluids Eng 131(2), (2009) 021303.

${ }^{[10]}$ P.F. Pelz, T. Keil, G. Ludwig, On the Kinematics of Sheet and Cloud Cavitation and Related Erosion. In Advanced Experimental and Numerical Techniques for Cavitation Erosion Prediction. Fluid Mechanics and Its Applications 106. Edts.: Kim, K.H., Chahine, G., Franc, J.P., Karimi, A., Springer 2014.

${ }^{11]}$ R. Fortes Patella, T. Choffat, J.L. Reboud, A. Archer, Mass loss simulation in cavitation erosion: Fatigue criterion approach, Wear 300(1-2), (2013), 205-215.

[12] A. Peters, H. Sagar, U. Lantermann, O. el Moctar, Numerical modelling and prediction of cavitation erosion, Wear 338-339, (2015) 189-201.
Article Title -7

${ }^{[13]}$ M. Dular, M. Petkovšek, On the mechanisms of cavitation erosion - Coupling high speed videos to damage patterns, Experimental Thermal and Fluid Science 68 (2015), 359-370.

$\left[{ }^{14]}\right.$ R. Garcia, F.G. Hammitt, Cavitation Damage and Correlations With Material and Fluid Properties, ASME J. Basic Eng 89(4) (1967) 753-763.

[15] S.G. Young, J.R. Johnston, Effect of temperature and pressure on cavitation damage to a cobalt base alloy in sodium, Characterization and Determination of Erosion Resistance, ASTM STP 474, ASTM, 1971, 67-102.

[16] M. S. Plesset, Temperature Effects in Cavitation Damage, ASME J. Basic Eng 94(3) (1972) 559-563.

${ }^{[17]}$ S. Hattori, I. Komoriya, S. Kawasaki, S. Kono, Cavitation erosion of silver plated coatings in a low-temperature environment, Wear 292-293 (2012) 74-81.

[18] S. Hattori, K. Taruya, K. Kikuta, H. Tomaru, Cavitation erosion of silver plated coatings considering thermodynamic effect, Wear 300(1-2)(2013) 136-142.

${ }^{[19]} \mathrm{C}$. Brennen, The dynamic behavior and compliance of a stream of cavitating bubbles, Transaction of the ASME (1973) 533-541.

${ }^{[20]}$ J.P. Franc, M. Riondet, A. Karimi, G.L. Chahine, Material and velocity effects on cavitation erosion pitting, Wear 274-275, (2012), 248-259.

${ }^{[21]}$ M. Gavaises, F. Villa, P. Koukouvinis, M. Marengo, J.P. Franc, Visualisation and les simulation of cavitation cloud formation and collapse in an axisymmetric geometry, International Journal of Multiphase Flow 68, (2015) 14-26.

${ }^{[22]}$ M. Dular, B. Širok, B. Stoffel, The influence of the gas content of water and the flow velocity on cavitation erosion aggressiveness. J. mech. eng. 51(3), (2005), 132-145.

[23 ]J.L. Reboud, R. Fortes-Patella, A. Archer. Analysis of damaged surfaces: Part I: Cavitation mark measurements by 3D laser profilometry. 3rd ASME/JSME Joint Fluids Engng. Conference, San Francisco, (1999).

${ }^{[24]}$ H. Lohrberg, M. Hofmann, G. Ludwig, B. Stoffel, Analysis of Damaged Surfaces and Part II: Pit Counting by 2D Optical Techniques. 3rd ASME/JSME Joint Fluids Engng. Conference, San Francisco, (1999), FEDSM99-6754.

${ }^{[25]}$ X. Escaler, M. Farhat, F. Avellan, E. Egusquiza, Cavitation Erosion Tests on a 2D Hydrofoil Using a Surface-Mounted Obstacles; Wear 254(5-6) (2003), 441-449.

[26] B. Bachert, G. Ludwig, B. Stoffel, S. Baumgarten, Comparison of Different Methods for the Evaluation of Cavitation Damaged Surfaces. ASME Fluid Engineering Division Summer Meeting and Exhibition, Houston, Texas, USA (2005), FEDSM2005-77368.

${ }^{[27]}$ P. F. Pelz, T. Keil, G. Ludwig, On the Kinematics of Sheet and Cloud Cavitation and Related Erosion, In Advanced Experimental and Numerical Techniques for Cavitation Erosion Prediction. Fluid Mechanics and Its Applications 106. Edts.: Kim, K.H., Chahine, G., Franc, J.P., Karimi, A., Springer 2014.

[28] A. Osterman, B. Bachert, B. Sirok, M. Dular, Time dependant measurements of cavitation damage, Wear 266 (910) (2009), 945-951.

[29] Y.C. Wang, C.E. Brennen, Shock wave development in the collapse of a cloud of bubbles. ASME FED Cavitation and Multiphase flow, 194 (1994). 


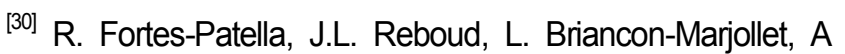
phenomenological and numerical model for scaling the flow aggressiveness in cavitation erosion. EROCAV Workshop, Val de Reuil, France (2004).

${ }^{[31]}$ G. Bark, N. Berchiche, M. Grekula, Application of principles for observation and analysis of eroding cavitation. The EROCAV observation handbook, Edition 3.1, (2004)

${ }^{[32]}$ G.L. Chahine, Modeling of Cavitation Dynamics and Interaction with Material. In Advanced Experimental and Numerical Techniques for Cavitation Erosion Prediction. Fluid Mechanics and Its Applications 106. Edts.: Kim, K.H., Chahine, G., Franc, J.P., Karimi, A., Springer 2014.

${ }^{[33]}$ M.S. Plesset, R.B. Chapman, Collapse of an innitially spherical vapour cavity in the neighbourhood of a solid boundary. J. Fluid Mech. 47 (1971) 283-290.

${ }^{[34]}$ M. Dular, B. Stoffel, B. Sirok, Development of a cavitation erosion model. Wear 261(5-6) (2006) 642-655.

[35] J.P. Franc, J.M. Michel, Fundamentals of Cavitation, Kluwer Academic Publishers (2004). 